\title{
Application of Computer Aided Design in Process Design of Acid Making by Flue Gas
}

\author{
Deng Gengsheng ${ }^{*}$, Zhang Guiping ${ }^{b}$ \\ a Network Center of Nanchang University, Nanchang, Jiangxi Province \\ ${ }^{b}$ Gongqing College of Nanchang University, Gongqing City, Jiangxi Province 332020 \\ *Corresponding Author: dgsheng@ncu.edu.cn
}

This is an open access article distributed under the Creative Commons Attribution License, which permits unrestricted use, distribution, and reproduction in any medium, provided the original work is properly cited

\section{ARTICLE DETAILS}

\section{Article History:}

Received 02 october 2017 Accepted 06 october 2017

Available online 11 october 2017

Keywords:

computer aided design; sulphuric acid; acid making process;

\section{ABSTRACT}

In order to liberate designers from complicated and repetitive calculation work, the design cost of sulphuric acid process is reduced. According to the production process of acid making by flue gas and the operation characteristics of computer program, the automation of design and calculation of acid making process is realized by using visual programming and database technology. This paper introduces how to apply the calculation process calculations of computerization, through sorting out the common formula, the basic calculation objects and links, data tables and other basic elements of common numerical calculation, model objects and links, and realize the function of the package and on this basis, the final realization of the calculation process program. The auxiliary calculation program has been continuously improved in the actual engineering projects, giving full play to the advantages of computer technology, greatly reducing the design cycle and improving work efficiency.

\section{Introduction}

In 2016, China's total output of sulfuric acid is $95.63 \mathrm{Mt}$, which was $1.1 \%$ lower than a year earlier; among them, the smelting flue gas production of acid is $33.13 \mathrm{Mt}$, accounting for $34.6 \%$ of total output, which increased by $9.6 \%$ year on year.In the future, in the case of smelting acid plant is still put into production, the elimination rate of backward production capacity will accelerate.[1]The design of an advanced sulphuric acid process which is consistent with the production capacity can effectively improve the production efficiency and reduce the environmental pollution hazards caused by sulfuric acid production.

The design of acid making process is limited by the output and the local conditions of the producers, which requires the design and selection of the corresponding manufacturing process and equipment for different production capacity and specific conditions .Process calculation is the precondition and necessary condition of sulphuric acid process design. The process calculation of flue gas acid production mainly includes process selection, material balance calculation, heat balance calculation and equipment selection. Each link influences each other. Traditional design has many problems, such as many parameters and large amount of calculation, resulting in long design cycle and low efficiency. In this paper ,the author illustrate how to realize the automatic calculation of acid making process design according to the sulphuric acid production process, combined with the characteristics of computer program, and through the use of visual programming and database technology.Actually, this method can greatly reduce the amount of calculation and the calculation error.

\section{Typical flue gas acid production process}

In a typical flue gas acid production process, purification, dry absorption and conversion are the three main stages ${ }^{[2]}$ :

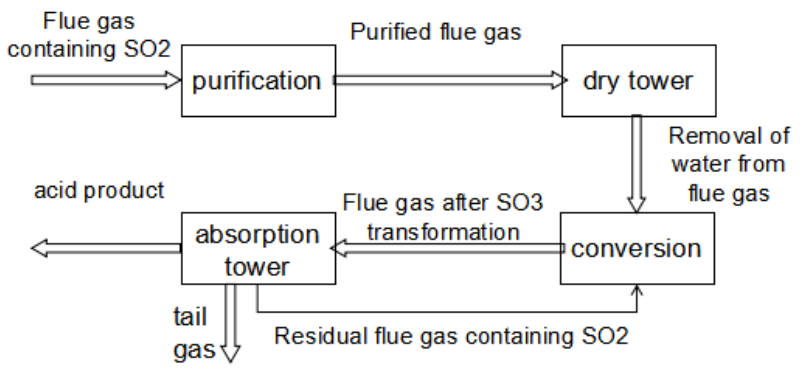

\section{Figure 1. Typical flue gas acid production process}

The flue gas from the pre-production section enters the acid production system, and the acid production system includes 3 processing sections, such as purification, drying, absorption and transformation. The flue gas enters the purification section first, removes the impurities such as smoke and dust in the flue gas, and then enters the drying tower in the drying section. It removes the moisture from the flue and enter the converter section in the dry tower.At one conversion, about $95 \%$ of the SO2 in the flue gas is converted to $\mathrm{SO}_{3} \quad\left(\mathrm{SO}_{2}+\frac{1}{2} \mathrm{O}_{2}=\mathrm{SO}_{3}\right)$, then it enters the intermediate absorption tower (the first absorption tower) absorbed in flue gas $\mathrm{SO}_{3}\left(\mathrm{SO}_{3}+\mathrm{H}_{2} \mathrm{O}=\mathrm{H}_{2} \mathrm{SO}_{4}\right.$, absorption by concentrated sulfuric acid ). The absorbed smoke continues to return to the conversion section for two transformations. The converted flue gas enters the final absorption tower (the second absorption tower) and absorbs the remaining $\mathrm{SO}_{3}$, which was discharged into the atmosphere by the chimney at last.

\section{Calculation in sulphuric acid manufacturing process design}

There are many calculation formulas of acid making process and they are complicated, whereas most of them are nonlinear variables. Many design parameters have a wide range of inquiries, so, we often need to calculate the difference, compare and adjust them repeatedly. In the process design of sulphuric acid production, material balance, heat balance and equipment process calculation should be carried out, and the process design and equipment selection should be carried out by the calculation results.

\subsection{Material balance calculation}

In the purification, conversion, dry suction and other sections, the total amount of material and smoke is equal to the total amount of output. Water balance and acid balance are included.

\subsection{Heat balance calculation}

Heat balance is to calculate the temperature of flue gas and acid water at different equipment according to the relevant conditions. It includes heat balance of cooling tower, heat balance of washing tower and heat balance of heat exchanger.

1) Heat balance of cooling tower : the cooling tower is adiabatic evaporation, so the cooling tower heat balance is the heat of the furnace gas intake and exit.

2) the heat balance of the washing tower: the heat balance of the washing tower includes the heat in and out of the boiler, the condensation of the water vapor and the heat of the acid out of the tower.

3) The heat balance of the heat exchanger is equal to the difference 
between the heat brought by the furnace gas and the heat brought out by the furnace gas.

\section{3 calculation of main equipment and process}

Calculation of all kinds of main equipment:

1) tower equipment: according to the basic conditions such as the amount of flue gas treatment, select the appropriate process parameters, equipment selection meter.

2) heat transfer equipment: according to the heat balance to get the equipment heat exchange, select the appropriate heat transfer coefficient,the heat exchange area is estimated preliminarily.

3) pump equipment: according to the tower type equipment to deal with the amount of flue gas, select the appropriate liquid gas ratio and calculate the pump specifications.

4) tank type equipment: according to the amount of circulating liquid, select the appropriate residence time and determine the groove type equipment specifications.

As the process of acid making involves many objects and links, it is complex and complicated, Repeated manual calculations require a lot of manpower and time, and can easily lead to errors. Therefore, in this paper, the computer aided process planning method is studied and a computer program is programmed to realize the auxiliary design of sulphuric acid process .It provides the electronic management and calculation service for the purification, drying, absorption and conversion of sulphuric acid manufacturing process, thus reducing the design cost, reducing the design cycle, and improving the work efficiency.

\section{Study on the application of computer aided calculation in the} process design of sulphuric acid

\section{1 computing requirements}

After the process design calculations and historical calculation of the case and data collation, the requirements of each link of the acid making process are as follows:

1) purification section calculation: By importing the imported smoke data, the pressure distribution of the equipment, the product acid concentration and other known data, the system automatically calculates the components of the exhaust gas, the total components of the aspirated gas, and the diameter of the equipment.

2) dry suction section calculation: By drying imported flue gas data, the known conditions and results in the calculation of the purification section, drying spray acid and absorption of the concentration of spray acid, temperature and so on, the system automatically calculate the gas amount and water amount on acid and absorption, and the diameter of the equipment.

3) conversion section calculation: According to the given flue gas composition and the heat exchanger flow, the temperature of each heat exchanger outlet can be calculated automatically. The conversion process includes single-absorption process and double transformation and double absorption process etc., according to different process double transformation and double absorption process is divided into III-I-IV-II, IV-I-III-II and so on, so in the design we should consider its scalability.

4) commonly used computing tools : In the process design, it is often necessary to calculate some of the specific links. Therefore, the design of heat exchangers, drying towers and absorption towers should be carried out independently.

\section{2 system module design and architecture}

According to the above analysis of the computer aided calculation needs, the system design is divided into six modules: system parameter management, purification section calculation, dry and dry section calculation, transformation section calculation, common calculation tools and custom conversion process. As shown in figure 2.

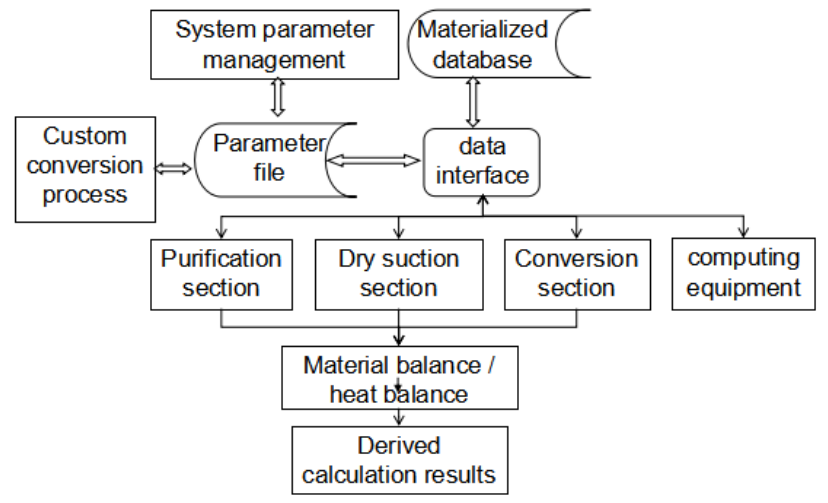

Figure 2. system architecture

Among them, the calculation of the purification section, the calculation of the transformation section and the calculation of the dry absorption section are the three main links in the acid making process, these three modules accept the input initial condition parameters.To obtain the required calculation data through the data interface from the established materialized database .Then, according to the system parameters that have been set, the results of material balance, heat balance and equipment selection are calculated. In the calculation process, the program will prompt for any error parameter settings that may occur in the process.

\subsection{Computerization of the process of calculation}

Process design calculation and historical calculation case is the basis information of calculation.In this paper, the basic calculation formulas, main calculation objects and links, data tables and common numerical values are sorted out by combing the basic data. According to different types of elements, the data are processed accordingly, so that they are implemented in a computer program. The process is shown in figure 3 :

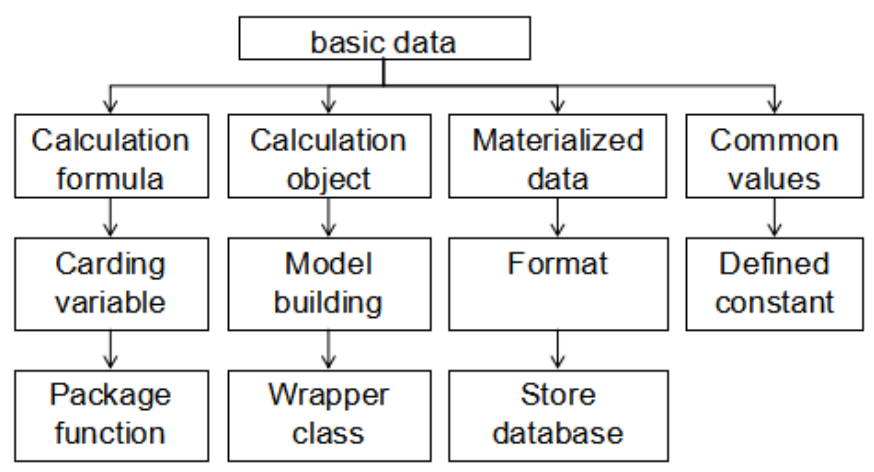

Figure 3. Computerized process of calculation

\subsection{Commonly used basic formula collation and function package}

The paper sorted out the common formula of the frequent use of the process in the existing design calculations, and they will be used for subsequent descriptions and implementation. Some commonly used basic formulas are listed below:

Basic formula 1 Gas heat content $Q=x \times C_{p} \times T_{p}$ coke / hour

In the formula, $\mathrm{X}$ is the amount of gas per hour (mol), Tp is the gas temperature, and the average molecular heat capacity (focal / molar) of the gas at $\mathrm{Cp}$ is $\mathrm{Tp}$.

Basic formula $2 \mathrm{H} 2 \mathrm{SO} 4$ dilute heat $Q=\left(\frac{17860 \times n}{n+1.7983}\right) \times 4.1863$ coke / mole In the formula, $\mathrm{Q}$ is the dilution heat, and $\mathrm{N}$ is the molar number of water used for $1 \mathrm{~mol}$.

Basic formula 3 T DEG C heat content of sulfuric acid $Q=W \times C_{p t} \times T$ The amount of $\mathrm{W}$ in water is $\mathrm{T}$, and CPT is the heat content of sulfuric acid. Basic formula 4 Interpolation method to calculate formula

$$
y_{3}=y_{1}+\frac{\left(x_{3}-x_{1}\right)\left(y_{2}-y_{1}\right)}{x_{2}-x_{1}}
$$

Among them $y_{2}, y_{1}, x_{2}, x_{1 \text { known }}$, 


$$
y_{2}>y_{3}>y_{1}, x_{2}>x_{3}>x_{1}
$$

\section{1) function encapsulation}

We encapsulate the base Formula 1 as a function, and the corresponding function is called formula_qtrhl $(\mathrm{x}, \mathrm{C}, \mathrm{t})$, this function completes the calculation of formula 1. Similarly, the function of the base formula 2 is called formula_lsxsr (n), and the base formula 3 has the function named formula_lsrhl (W, $\mathrm{C}, \mathrm{t}$ ). As the function package is completed, when other calculations need to use these formulas, we can invoke them by function.

\subsection{Establishment and calculation method of materialized database}

Physical properties of many compounds are often used in the calculation of sulphuric acid process ,such as density, vapor pressure, heat capacity, heat content, reaction heat, etc..The initial data provided only include the basic materialized data, but for actual calculations, if the direct lookup is not available, the calculation of the materialized data is needed. Considering the extensibility of the software, the design uses the database to store materialized data, and enriches the materialized data in the process of actual use. When the next materialized data is calculated again, it can be directly searched and obtained. The calculation of unknown physical data is calculated by interpolation method, such as basic formula 4. By finding the data of the upper and lower region nodes of the data to be obtained, the data distribution in this region is assumed linearly. According to the distance between the point to be solved and the upper and lower nodes, the data of the upper and lower nodes is substituted, and the materialized data of the point to be searched is obtained.

\subsection{To establish the calculation model of main calculation objects and links}

In this section, a computational model is established for the main targets and links in the process of acid production. Several typical objects are listed below, and their computational models are briefly explained.

1) gas.Generally speaking, the weight, volume, molar number, temperature, pressure, etc. of the gas should be set to calculate the heat content or temperature. Relevant parameters: molecular weight, volume, weight, pressure, temperature, heat content, etc.; calculation requirements: according to the basic formula 1 to calculate the heat content, weight volume mole number conversion;

2) acid. Generally speaking, the concentration, temperature, volume, weight, etc. of the acid should be set to calculate the heat content or temperature. Relevant parameters: temperature, concentration, flow rate, weight, volume, heat content, etc.; calculation requirements: according to the basic formula 3 , calculate heat content, calculation temperature, weight volume flow rate conversion;

3) mixed gas. In actual calculation, the gases that pass in and out are mixed gases, including $\mathrm{SO}_{2}, \mathrm{SO}_{3}, \mathrm{O}_{2}, \mathrm{~N}_{2}, \mathrm{H}_{2} \mathrm{O}$, CO, etc.. Relevant parameters: $\mathrm{SO}_{2}, \mathrm{SO}_{3}, \mathrm{O}_{2}, \mathrm{~N}_{2}, \mathrm{H}_{2} \mathrm{O}$, CO, and volume, weight, pressure, temperature and heat content of gas type. Calculation requirement: heat content is calculated according to formula 1 ;

4) absorption tower. Usually, $98 \%$ of the absorbed acid absorbs SO3, and the concentration increases to $98 . \mathrm{xx} \%$, so the temperature of the tower acid should be calculated. Relevant parameters: inlet acid, outlet acid, inlet tone, breath, liquid gas ratio, absorption rate, conversion rate, pressure difference between inlet and outlet, temperature difference between inlet and outlet; Calculation requirement: the heat content of tower acid is calculated according to the basic formula 2 and the basic formula 3 , and the tower acid concentration, tower acid temperature and reaction heat are calculated:

\subsection{Class encapsulation of computational models}

When the program is implemented, the computing model generated by the object is encapsulated into a corresponding class, Where the calculation involves the input and output parameters as the object's properties, the computational process is encapsulated into a method. We first define the most basic gas, water, acid, circulation tanks, drying towers, absorption towers, acid coolers, scrubbers, heat exchangers and so on, the gas mixture, the dry absorption tower and the like are then packed. encapsulating an object model into a class:

First, Let's define the AbsorbTower class. The related parameters such as acid, acid, into export into the tone, tone, liquid gas ratio, absorption rate, conversion rate, exit pressure, entrance temperature variation are defined as the attributes of the class. Among them, the type of inlet acid and outlet acid are acids, the inlet gas and the outlet gas are mixed gases. The calculation of the heat content of the tower and the calculation of the concentration of the tower acid, the temperature of the tower and the reaction heat are defined as the methods of this kind. Here is part of the code example:
Private mvaracid_IN As Acid
Private mvaracid_OUT As Acid
Private mvargas_IN As MixGas
Private mvargas_IN_2 As MixGas
Private mvargas_OUT As MixGas
Private mvaryqb As Double
Private mvarZHL As Double
Private mvarXSL As Double
Private mvarpressurediff As Double
Private mvartemperaturediff As Double
Public Function count_SNCO As Double
Public Function count_Q_Reaction0 As Double
Public Function count_Q_Acid_Out0 As Double
Public Function count_TSC() As Double

\section{System implementation effect}

The software uses Microsoft Visual Studio.NET member VB.NET as the development platform,the graphical user interface (GUI) design is adopted, which is easy to operate and use.The database uses Microsoft Access, which does not require the user to install the database server, and can be used directly to drive, and can meet the substantial needs of materialized data expansion.The main functions of the software include file management, system parameter setting, cleaning section calculation, dry suction section calculation, custom conversion process and common calculation tools. The common calculation tools include independent calculation of cooling towers, heat exchangers, drying towers and absorption towers.

This software has been continuously modified and improved in the process of testing and verification since 2010 . It is now more mature and reliable, which plays a very important role in the design work. For projects of large size and complicated design conditions, From the process of design to the stage of basic design, the project plan may be changed several times. Among them, only the catalyst scheme of the conversion section is as many as ten kinds Relying on traditional manual computing you will undoubtedly need a lot of time. In the design process of the project, the author uses sulfuric acid process calculation software to calculate, so as to deal with a lot of computing content quickly.The verification proves that the calculation result is accurate and reliable.

\subsection{Test using sample}

In the process design of sulphuric acid production, the process and calculation of the conversion section are more flexible, Therefore, this paper takes the calculation of conversion section as an example to introduce the test and use of software.

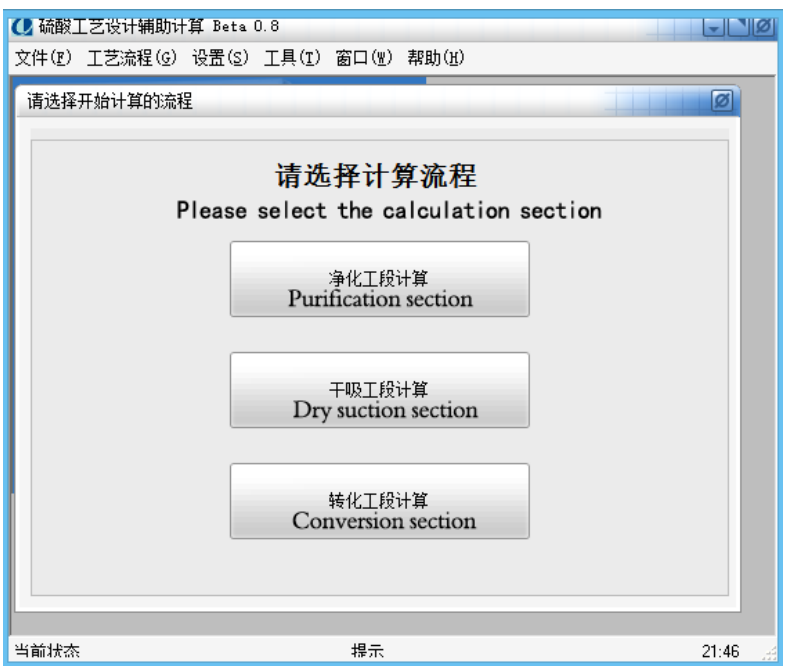




\section{Figure 4. Screenshot of software interface}

In order to select the heat exchanger better, the customization function of the conversion section is designed in the software, we can select the number of heat exchangers, order, dry suction inlet position, unknown port, etc., As shown in Figure 5, we created the conversion process of "3142".

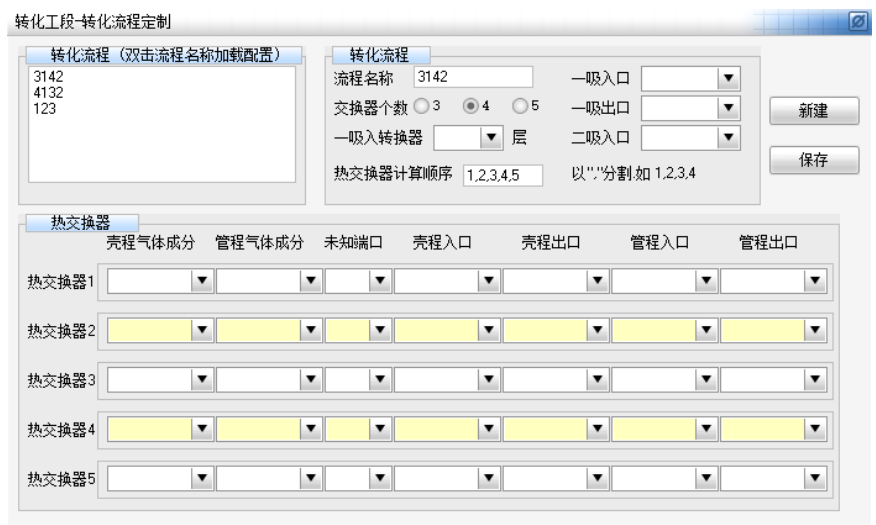

Figure 5. custom conversion process interface

If you enter into the conversion section of the calculation interface, you can see that the creation of "3142" conversion process is displayed on the interface, When we set the initial conditions of the calculation, we can choose the "3142" conversion process to calculate.

Click the "calculate" to get the calculation results, as shown in Figure 6, you can export the results of calculation.

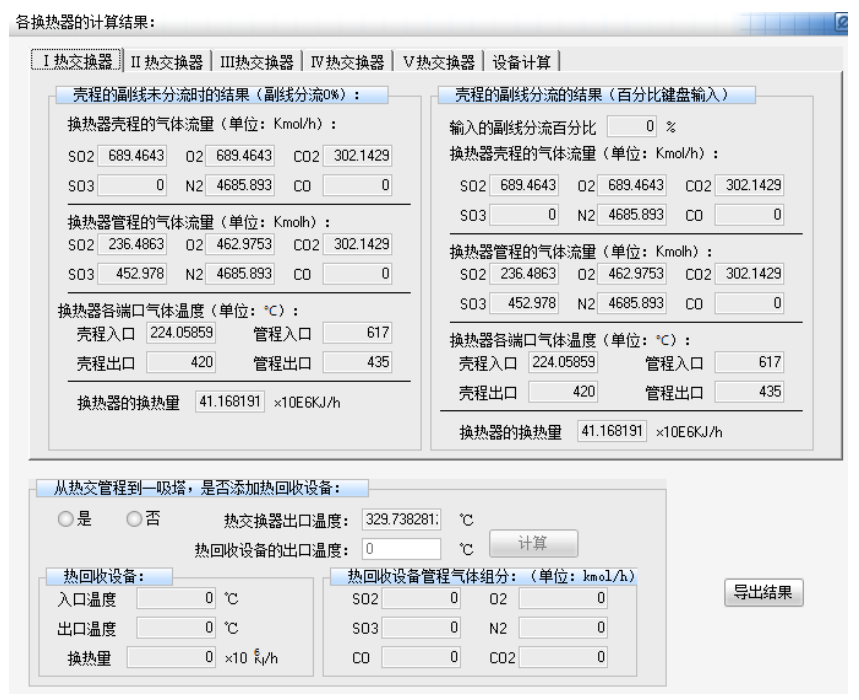

5 Conclusion

This paper introduces the typical process of acid production by flue gas The calculation contents in acid process design are listed, and the demand of computer aided calculation in acid making process is analyzed. According to the requirement, the system structure and module of computer aided process planning are designed. According to the working characteristics of computer program, the automation of design and calculation of acid making process is realized by using visual programming and database technology. This paper introduces how to computerize the design calculations and the computing process in the history case.By sorting out the basic commonly used formulas, main calculation objects and links, data tables and common values, and other basic elements, a computational model for calculating objects and links is established. On this basis, the encapsulation of functions and classes is realized, and finally the whole computing process is programmed. With the actual engineering project, the auxiliary calculation program is constantly modified and improved in the process of testing and verification. The user only needs to input the initial parameters and click the mouse to get the desired results. The results of detailed computational objects can be derived as required .The application of software liberates designers from complex and repetitive computing efforts .It can effectively reduce the cost of sulphuric acid process design, reduce the design cycle and improve work efficiency. Because the program is based on the original statement and historical case design , the intelligence of the software is not enough. In some calculation links, some parameters should be adjusted and corrected artificially, which will be improved and improved in the future work.

\section{Reference}

[1] Chong, L. I., \& Liao, K. (2017). Production of sulfuric acid industry in 2016 and prospect in 2017 in china. Phosphate \& Compound Fertilizer.

[2] GuoKai, Huang weihua, \& Caoxia. (2014). Design and Realization of Sulfuric Acid Process Calculation Software. Nonferrous Metals Engineering \& Research(5), 46-49.

[3] Wang lijun, \& Zhou fang. (2016). The Function of Computer Aided Design in Chemical Process. Chenmical Intermediate(3), 64-65.

Kong, J. (2008). Visual language techniques for software development visual language techniques for software development. Journal of Software 19(8), 1902-1919.

[4] Kiss, A. A., Bildea, C. S., \& Grievink, J. (2010). Dynamic modeling and process optimization of an industrial sulfuric acid plant. Chemical Engineering Journal, 158(2), 241-249.

\section{About the Authors}

Deng Gengsheng (1980 -), male, Ji'an, Jiangxi, China, engineer, master, research direction: computer application and network.

Zhang Guiping (1984 -),female,Yongxiu,Jiangxi,China,Lecturer, master, English teaching and interpreting.

Figure 6. calculation result of conversion section

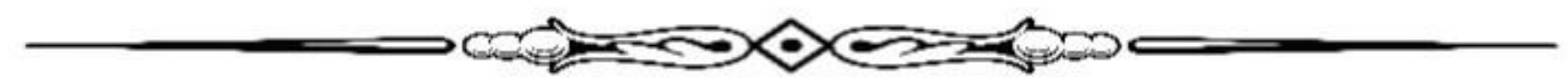

